\title{
MASUMI NAGASAKA
}

\section{ÜBER DEN BEWEIS DES TRANSZENDENTALEN IDEALISMUS BEI HUSSERL — AUS DEN TEXTEN Nr. 5-7 IN HUSSERLIANA XXXVI"}

\begin{abstract}
About Argument of Transcendental Idealism on Husserl: Texts No. 5-7 (Husserliana XXXVI)

The aim of this paper is to show the mutual dependence between Husserlian concepts of the «idea in the Kantian sense» and the principle of contradiction, through the reading of Husserl's texts concerning the «proof (Beweis) of transcendental idealism» from 1913 to 1915.

Keywords: idea in the Kantian sense, real possibility, merely ideal possibility, displacement (Aufschub), law of contradiction, reality, transcendental idealism.

ОБ АРГУМЕНТЕ ТРАНСЦЕНДЕНТАЛЬНОГО ИДЕАЛИЗМА ПО ГУССЕРЛЮ: ТЕКСТЫ № 5-7 (ГУССЕРЛИАНА XXXVI)

Статья посвящена демонстрации взаимозависимости гуссерлевского понятия «идеи в кантовском смысле» и принципа противоречия посредством анализа текстов Гуссерля 1913 1915 гг., в которых разбирается «аргумент трансцендентального идеализма».

Ключевые слова: идея в кантовском смысле, реальная возможность, только лишь идеальная возможность, перенос (сдвиг), закон противоречия, действительность, трансцендентальный идеализм.
\end{abstract}

\section{EINFÜHRUNG}

$\mathrm{D}$ ieser Aufsatz fokussiert den Husserlschen Beweis des transzendentalen Idealismus, der in den Texten Nr. 5 bis Nr. 7 in Husserliana XXXVI entwickelt wurde. ${ }^{1}$ Im Grunde genommen nennt man die einseitige Abhängigkeit der Welt vom Bewusstsein einer Subjektivität Idealismus, wo-

* Eine verkürzte und dazu auf die Lektüre Husserls durch Derrida eingehende erheblich umstrukturierte Ausgabe dieses Aufsatzes wurde am 3. Juli 2009 unter dem Titel «Über den Aufschub in der Idee im Kantischen Sinne und den Satz vom Widerspruch bei Husserl» an der Universität Wuppertal bei der Tagung «Grundprobleme der phänomenologischen Erfahrung» vorgetragen, die von der Studentenvereinigung Amical des MasterErasmus-Mundus EuroPhilosophie organisiert wurde.

1 Husserl E. Transzendentaler Idealismus, Texte aus dem Nachlass (1908-1921) / hrsg. von Robin D. Rollinger in Verbindung mit Rochus Sowa. Husserliana (Hua) XXXVI. Dordrecht; Boston; London: Kluwer Academic, 2003. S. 73-145. Der Text Nr. 5 stammt aus der Vorlesung «Natur und Geist» des Sommersemesters 1913, der Text Nr. 6 aus der Vorlesung «Ausgewählte phänomenologische Probleme» des Sommersemesters 1915. Der Text Nr. 7 datiert nach dem Herausgeber in 1914 oder 1915. Die Zitate aus dieser Ausgabe werden im Folgenden mit der Abkürzung Hua XXXVI gekennzeichnet. In allen Zitaten gehören die Kursivierungen zu den originalen Texten.

(C) M. Nagasaka, 2012 
bei das Bewusstsein seinerseits unabhängig von der Welt gedacht wird. Der Husserlsche «transzendentale» Idealismus muss aber noch in dem Sinne weiter präzisiert werden, dass dieser die klassischen Positionen des Idealismus und Realismus nicht ausschließt, sondern beide als «denkbar» erklären kann. Der Realismus, der die Welt ohne Bewusstsein ansetzt, und der Idealismus, der das Bewusstsein ohne Welt ansetzt, sind beide wohl «denkbar», aber der transzendentale Idealismus behandelt beide nicht als ausschließliche Alternativen.

Einerseits ist die Welt ohne Bewusstsein durch ein aktuell erfahrendes Bewusstsein denkbar, wenn die ohne Bewusstsein existierende Welt nachträglich, d. i. «rückwärts», als rekonstituierte «Vergangenheit» ${ }^{2}$ ausgewiesen wird: «Es muss gezeigt werden, dass nur wenn wirkliche Subjekte in wirklichen Erfahrungen eine Natur bewusstseinsmäßig sich gegenüber haben können, die Existenz einer Welt ohne Menschen (Vernunftwesen, hier: erfahrende Wesen) denkbar ist. Eine Welt ohne Subjekte, die wirklich sie erfahren $<_{\ldots} .$. , ist nur denkbar als Vergangenheit einer Welt mit solchen Subjekten»». ${ }^{3}$

Damit die Welt ohne Bewusstsein existieren kann, muss sie als solche ausgewiesen werden, d.h. sie muss durch ein aktuell erfahrendes Bewusstsein, das nicht notwendig gegenwärtig sein muss, nachträglich konstituiert werden. Anderseits ist das Bewusstsein ohne Welt wohl denkbar durch die Unabhängigkeit des Bewusstseins von der Welt.

Die Eigentümlichkeit des Beweises des transzendentalen Idealismus in Husserliana XXXVI besteht darin, dass Husserl hier die Unterscheidung zwischen der «bloß idealen Möglichkeit», die von der Wirklichkeit vollkommen frei ist, und der «Wirklichkeit» oder der «realen Möglichkeit», die mit dieser Wirklichkeit verbunden ist, einführt. Unter Berücksichtigung dieser Unterscheidung wird die Abhängigkeit der Welt vom Bewusstsein im Husserlschen Sinne so präzisiert, dass die mögliche Welt das wirkliche Bewusstsein voraussetzt, und die Unabhängigkeit des Bewusstseins von der Welt wird so präzisiert, dass die Welt möglich sein kann, ohne wirklich zu sein, während das Bewusstsein notwendig wirklich existieren muss.

2 «Eine bloß materielle Welt als Unterstufe und als Anfangsstrecke der Dauer der Welt genügt den Bedingungen der Erkennbarkeit, wenn eine Subjektivität existiert, die $<\ldots>$ vernunftgemäß [die vorangegangenen Weltstrecken] rückwärts konstruieren kann...» (Hua XXXVI. S. 141).

3 Hua XXXVI. S. 144, Fußnote 2. 
Der erste Punkt dieser These des transzendentalen Idealismus wird wie folgt von Husserl beschrieben: «Die Möglichkeit der Natur setzt voraus oder schließt idealiter ein die Möglichkeit einer Erkenntnis dieser Natur. Die letztere Möglichkeit fordert, das wäre dann weiter zu zeigen, nicht die bloß logische Möglichkeit von „Menschen in der Natur“, sondern wirkliche Existenz derselben». ${ }^{4}$

Die Aufgabe, mit der sich dieser Aufsatz beschäftigen wird, ist es, den Beweis für diesen Punkt näher zu beleuchten und zu diskutieren. Der zweite Punkt bezieht sich auf die Möglichkeit der «Vernichtung der Welt». Husserl versucht zu zeigen, «dass die Welt überhaupt nicht zu sein braucht». ${ }^{5}$ Dies werden wir ebenfalls näher betrachten.

Durch die Lektüre dieses Beweises wird das grundlegende Prinzip der Husserlschen Phänomenologie, nämlich die Zusammengehörigkeit der Einheit und der Gemeinsamkeit der Zusammenhänge der Wirklichkeiten, abgeleitet werden. Daraus folgt die Problematik bezüglich des Husserlschen Begriffs der sogenannten «Idee im Kantischen Sinne» und des Satzes vom Widerspruch. Diesbezüglich werden sich Fragen ergeben, die im Schlusskapitel diskutiert werden, in dem Derridas Lektüre Husserls berücksichtigt wird.

\section{Die Vorstufe für den Beweis des tRanszendentalen Idealismus}

\section{DAS WESEN DER WAHRNEHMUNG}

Bevor wir mit unserem Thema beginnen, soll die Voraussetzung für den Beweis ins Auge gefasst werden. Von $\S 1$ bis $\S 8$ im Text Nr. 6 wiederholt Husserl seine wichtigen Grundgedanken über die Wahrnehmung, die in Ideen I entwickelt wurden. Davon werden wir unter anderem, erstens, das Wesen der Wahrnehmung - als verschiedene Apriori, die zur Wahrnehmung gehören -, wo das Wahrgenommene als originär Gebendes sich gibt, beobachten. Zweitens wird gefragt werden, warum dieses Wahrgenommene als originär Gegebenes aufgefasst werden muss bzw. warum es nicht als ein ähnliches Bild oder ein Zeichen von «an sich seiendem» Anderen genommen werden darf.

\section{1) Verschiedene Apriori der Wahrnehmung}

Zuerst werden wir die verschiedenen Apriori kennenlernen, die zum Wesen der Wahrnehmung gehören, beispielsweise die Orientierung, um Gegenständ-

4 Hua XXXVI. S. 135.

5 Ibid. S. 112. 
lichkeiten wahrzunehmen, die damit verbundene Leiblichkeit des Wahrnehmenden, ihre Freiheit in der Räumlichkeit. Husserl erweitert den Begriff von «a priori», um ihn für das materielle, konkrete, transzendente Wahrgenommene verwenden zu können. Betrachten wir unter anderem die Apriorität der Untrennbarkeit der Farbe und der Ausdehnung: «Im wahrgenommenen Realen ist die gegebene Ausdehnung zwar faktisch mit den bestimmten sinnlichen Qualitäten und keinen anderen behaftet, aber darin waltet ein Apriori. Ausdehnung ohne Färbung und sonstige Sinnesqualität ist schlechthin undenkbar, ein Nonsens». ${ }^{6}$

Außer diesem Apriori werden verschiedene andere Apriori erwähnt. Beispielsweise ist mit transzendentem Wahrgenommenen, das sich durch verschiedene Abschattungen ergibt, a priori die Veränderung der Aspekte in der Orientierung im doppelten Sinne verbunden, nämlich in ihrer «zeitlichen und räumlichen Orientierung». ${ }^{7}$ Husserl sagt: "[Es] ist immer zu $<$ be $>$ achten, dass Orientierung und sich abschattende Erscheinung unabtrennbar zusammengehören und dass die Beziehung auf die Orientierung jeder Erscheinungskomponente in gleicher Weise anhängt». ${ }^{8}$ Das Wahrgenommene, das abschattend erscheint, braucht notwendig die Orientierung des Wahrnehmenden. Diese Orientierung ist einerseits zeitlich und andererseits räumlich durch den Wahrnehmenden bestimmt. ${ }^{9}$ Es muss aber beachtet werden, dass die zeitliche Orientierung keiner Willkür des Wahrnehmenden ausgesetzt ist, während die räumliche Orientierung von der Freiheit subjektiver Willkür abhängt.

Diese Apriorität der Orientierung ist durch die Apriorität der Möglichkeit der Bewegung, der Leiblichkeit ${ }^{10}$ und der Fähigkeit, ${ }^{11}$ verschiedene räumliche Stellungen einzunehmen, vorgezeichnet. Die Orientierung gehört zum Wesen der Wahrnehmung und nicht zur Menschheit, die etwa im Vergleich zu Gott

6 Hua XXXVI. S. 88.

7 Ibid. S. 133.

8 Ibid. S. 95.

9 «Die zeitliche Orientierung ist eine während der Erfahrung fließende, nicht freie, keiner Willkür des Subjekts unterliegende. Die räumliche hingegen unterliegt nach Unveränderung oder Veränderung $<\ldots>$ der Freiheit und Willkür...» (Hua XXXVI. S. 133).

10 «...es [ist] a priori notwendig $<\ldots>$, dass das erfahrende Ich für seine freie Stellungsänderung eines frei beweglichen und ihm selbst räumlich erscheinenden Leibes bedarf» (Ibid.).

11 «Ich, der Erfahrende, "kann" meine "Stellung" zu den Dingen und damit zur ganzen Erfahrungswelt bzw. "in” ihr ändern» (Ibid.). 
beschränkt wäre: Auch wenn Gott allmächtig ist, bräuchte er eine Orientierung für die Wahrnehmung. ${ }^{12}$

Diese räumliche und zeitliche Orientierung, um Gegenständlichkeit wahrnehmen zu können, bedeutet, dass sich das Wahrgenommene in der Kontinuation ${ }^{13}$ der Wahrnehmung als Kontinuum zeigt, das seine Einheit und Einstimmigkeit hat, die aber immer unvollkommen bleiben. Der Wahrnehmung kommt eine Einstimmigkeit zu, der immer eine Zurücknahme offenbleibt. In dieser Kontinuation ist das Wahrgenommene «nicht beliebig», sondern in der Fortsetzung der Wahrnehmung «vorgezeichnet» ${ }^{14}$.

\section{2) Die Kritik der Bilder- und Zeichentheorie}

Das, was wahrgenommen wird, ist originär gebenden. Hier sollte die Husserlsche Kritik der Bilder- und Zeichentheorie beachtet werden, die Husserl in $\S 43$ in Ideen I entwickelt, ${ }^{15}$ aber in $\S 6$ im Text Nr. 6 einleuchtender erklärt: Die Bilder- und Zeichentheorie kann den «unendlichen Regress $»{ }^{16}$ nicht vermeiden.

Die Bilder- und Zeichentheorie behauptet Folgendes: «Es treten in meinem Bewusstsein Bilder oder Zeichen auf». ${ }^{17}$ Diese Theorie stellt sich das Bewusstsein wie einen «Kasten» vor: «Man stellt sich da so ungefähr das Bewusstsein wie einen Kasten vor, worin den äußeren Dingen ähnliche Objekte, abbildliche Objekte, sind, wobei man zu fragen vergisst, wie nun das Ich zu denken ist, welches die Abbilder als solche der äußeren Dinge erfasst und erkennt». ${ }^{18}$

Wenn man so denkt, muss man ein anderes Ich voraussetzen, das das Objekt außerhalb des Kastens und das Bild dieses Objekts im Kasten vergleichen kann. In einer solchen Vorstellung liegt das Problem des klassischen Realis-

12 «Es [= hier ist die Orientierung gemeint] ist nicht Sache des Menschen, sondern Sache des eigenen Wesens der Wahrnehmung und ihres Wahrgenommenen als solchen» (Hua XXXVI. S. 93).

13 «Zum Wesen der Wahrnehmung also gehört, dass sie in noch so weit geführter Kontinuation offen lässt, <...> (Hua XXXVI. S. 98).

14 Ibid.

15 Husserl E. Ideen zu einer reinen Phänomenologie und phänomenologischen Philosophie. Hua III/1, Den Haag: Nijhoff, 1976. S. 89 ff. Die Zitate aus dieser Ausgabe werden im Folgenden mit der Abkürzung Hua III/1 gekennzeichnet.

16 Hua XXXVI. S. 107.

17 Ibid. S. 106.

18 Ibid. 
mus und Idealismus. So ergäbe sich ein in zwei verschiedene Standpunkte gespaltenes Ich. Aber um dieses Bild im Kasten zu sehen, müsste es einen anderen weiteren Kasten geben, darin das gespaltene Ich das Bild im ersten Kasten und das Bild von diesem Bild im zweiten Kasten vergleichen kann. ${ }^{19}$ So müsste sich das Ich in infinitum spalten. «Das Ich ist doch nicht ein kleines Menschlein im Kasten darin, das sich die Bilder ansieht und dann gelegentlich einmal aus dem Kasten herauskriecht und die Dinge draußen mit denen drinnen vergleicht usw. Für dieses das Bild sehende Ich wäre ja das Bild wieder ein Draußen, für welches ein Bild drinnen in seinem Bewusstsein gesucht werden müsste, und so in infinitum». ${ }^{20}$

Also muss das Wahrgenommene als originär Gegebenes bestimmt werden: «In der Wahrnehmung haben wir nicht ein Bild vor Augen, sondern wir haben die Sache selbst vor Augen. Das ist der eigene Sinn der Wahrnehmung, das gehört zu ihrem Wesen...». ${ }^{21}$ Es wäre widersinnig, sich etwa ein «mystisch an sich Seiendes», «Unzugängliches ${ }^{22}$ vorzustellen.

\section{DIE REALE MÖGLICHKEIT / DIE WIRKLICHKEIT UND DIE BLOß IDEALE MÖGLICHKEIT}

Wie in der Einführung erwähnt, ist die Unterscheidung zwischen realer Möglichkeit und bloß idealer Möglichkeit für diesen Beweis äußerst wichtig. Husserl führt die Unterscheidung zwischen Wirklichkeit und bloß idealer Möglichkeit im Text Nr. 5 ein: «Wir unterscheiden die Wirklichkeit des Dinges von seiner bloß idealen Möglichkeit». ${ }^{23}$ Noch wichtiger aber ist die Unterscheidung zwischen der realen Möglichkeit, die mit der Wirklichkeit verbunden ist, und der bloß idealen Möglichkeit. Wir werden das, die folgenden vier Punkte beachtend, darstellen: das Recht, die Regel, die Habitualität und die Ausschließlichkeit.

\section{1) Das Recht der realen Möglichkeit}

Zuerst muss beachtet werden, dass die reale Möglichkeit ein von der Wirklichkeit motiviertes, bekräftigtes Recht hat. Husserl spricht von Rechtsgründen «primärer» und «sekundärer» Stufe: «Jede Erfahrung hat ja evidenterwei-

\footnotetext{
19 Siehe Abbildung 1.

20 Hua XXXVI. S. 106.

21 Ibid. S. 107.

22 Ibid.

23 Ibid. S. 75.
} 
se ein Recht an sich. $<\ldots>$ Dabei hat die Wahrnehmung einen primären und vorzüglichen Rechtsgrund hinsichtlich des eigentlich Erfahrenen, $<\ldots>$. Aber ein sekundäres Recht, das immer noch Recht ist, reicht doch weiter. Wie die Rückseite aussieht, das ist in der einseitigen Wahrnehmung nicht gegeben, aber die Wahrnehmung motiviert doch in sich selbst die Annahme einer gewissen, wenn auch unvollkommen bestimmten und nicht anschaulich gegebenen Rückseite: $<\ldots>\gg{ }^{24}$

Während das primäre Recht in der aktuellen Wahrnehmung liegt, wird das sekundäre Recht durch diese Wahrnehmung motiviert. Beispielsweise ist die Rückseite des Wahrgenommenen zwar «unbestimmt», aber es ist «eine bestimmbare Unbestimmtheit», sie ist schon mit dem Wahrgenommenen «mitgesetzt» und hat ihren sekundären «Rechtsanspruch, der nicht leer ist, der seine Kraft hat». ${ }^{25}$ Das schließt nicht aus, dass diese Kraft im Fortgang der Wahrnehmung nachträglich widerrufen werden kann.

Damit könnte der Unterschied von der realen Möglichkeit und der bloß idealen eingesehen werden. Die reale Möglichkeit ist die Möglichkeit, die dieses sekundäre Recht hat, die das reale Mögliche «zur Aktualisierung» ${ }^{26}$ kommen lassen kann, während die bloß ideale Möglichkeit diejenige ist, die dieses Recht nicht hat. Zwar fordert die bloß ideale Möglichkeit auch die Einstimmigkeit, insofern sie, als die Quasi-Wahrnehmung in der Fiktion beispielsweise, ein Kontinuum konstituiert, aber diese Einstimmigkeit ist nicht mit der Wirklichkeit verbunden, mit anderen Worten, sie hat keine Konvergenz in Bezug auf die Wirklichkeit, sie ist frei von ihr.

\section{2) Die Regel, die die Kantische regulative Idee vorschreibt}

Das bezieht sich auf die Regel, die die reale Möglichkeit hat. Die Verbundenheit der realen Möglichkeit mit der Wirklichkeit ergibt diese Regel, die die reale Möglichkeit auf eine schmalere Breite beschränkt, während die bloß ideale Möglichkeit, die von jeder Regel frei bleibt, eine viel größere Breite hat. Die Regel, mit der die reale Möglichkeit verbunden ist, könnte als eine konvergente Reihe angesehen werden, die auf die Wirklichkeit abzielt.

In diesem Sinne ist die reale Möglichkeit — so präzisiert Husserl — mit der regulativen Idee im Kantischen Sinne verbunden: «Die Möglichkeiten der Erfahrung, die aus diesen aktuellen, an wirklichen Gegebenheiten verankerten

24 Hua XXXVI. S. 118.

25 Ibid.

26 Ibid.

$70 \quad$ Masumi Nagasaka 
Motivationen entspringen, sind dann nicht mehr rein ideal und völlig frei, sondern durch Seinsthesen gebundene Möglichkeiten. Freilich, die Existenz des Dinges ist für das aktuelle Bewusstsein immerfort eine Idee, aber eine Idee $<\ldots>$ im Kantischen Sinn, wir könnten sogar das Wort von der regulativen Idee verwenden $\left\langle\ldots>\gg .{ }^{27}\right.$ Hier verwendet Husserl seine These über das «Ding» als eine «Idee im Kantischen Sinne», die in Ideen $I, \S 143$ dargestellt wurde. ${ }^{28}$ Die reale Möglichkeit steht im Horizont der «wirklichen Gegebenheiten», auf die sie aufgewiesen ist. Mit anderen Worten weist Husserl an dieser Stelle implizit darauf hin, dass die Wirklichkeit nichts anderes als eine Idee im Kantischen Sinne ist, die der realen Möglichkeit eine Regel gibt. Dagegen ist die bloß ideale Möglichkeit keine Regel, die von der regulativen Idee vorgeschrieben wird.

\section{3) Die Habitualität des Fortgangs der Erfahrung}

In diesem dritten Punkt soll festgestellt werden, dass diese reale Möglichkeit mit der «Habitualität», d. h. der «Selbstbewährung» ${ }^{29}$ des Kontinuums, mit der gewöhnlichen Wirklichkeit verbunden ist. Durch diese Habitualität wird die reale Möglichkeit im möglichen synthetischen Fortgang der Wahrnehmung antizipiert: «Die Welt ist vor der Wissenschaft mir und jedermann ursprünglich gegeben durch sinnliche Wahrnehmung, und sie ist Welt der habituellen Erfahrung, die bleibend seiende Welt, aus der Habitualität der schon gestifteten Erfahrungsgeltung vorgegebene Welt». ${ }^{30}$ Die reale Möglichkeit wird so aufgefasst, dass sie schon mit der Habitualität der bleibend seienden Welt verbunden ist, während die bloß ideale Möglichkeit von der Habitualität viel freier bleibt.

4) Die Ausschließung der realen Möglichkeiten in ihrer Aktualisierung von der Wirklichkeit

Zuletzt soll beobachtet werden, dass die reale Möglichkeit in ihrer Aktualisierung durch die Wirklichkeit ausgeschlossen werden kann. Das «Ding», das als die Wirklichkeit existiert, ist keine bloß ideale Möglichkeit, dazu gehört die Ausschließung gegenüber seinen anderen realen Möglichkeiten. Das Ding kann nichts anderes, denn als solches in der Wirklichkeit existieren. Dagegen hat die bloß ideale Möglichkeit, beispielsweise ein Zentaur, nicht diese Ausschließlichkeit, sondern die unendliche Freiheit, wo unverträgliche verschiede-

27

28

29

30

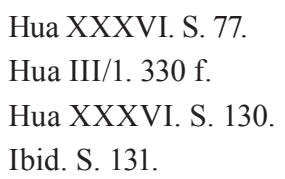

Horizon 1 (2) 2012 
ne Formen einander nicht ausschließen: «Ich phantasierte mir den Zentauren blond und zweiäugig, ich kann mir ihn ebenso gut schwarzhaarig und einäugig vorstellen, obschon die eine ideale Möglichkeit mit der anderen unverträglich ist». ${ }^{31}$ So könnte gesagt werden, «...dass die bloß ideale Möglichkeit eines Dinges, z. B. eines Zentauren, nicht unendlich viele andere, miteinander in der Existenz unverträgliche ideale Möglichkeiten ausschließt». ${ }^{32}$

So wird die reale Möglichkeit dadurch von der bloß idealen Möglichkeit unterschieden, dass sie von der Wirklichkeit ausgeschlossen werden kann, während die bloß ideale Möglichkeit von der Wirklichkeit nie ausgeschlossen werden kann.

Hier wurde die Unterscheidung zwischen der mit der Wirklichkeit verbundenen realen Möglichkeit und der bloß idealen Möglichkeit durch das Recht, die Regel, die Habitualität und die Ausschließlichkeit der Wirklichkeit betrachtet. Diese Unterscheidung wird eine wichtige Rolle im Beweis des transzendentalen Idealismus spielen.

\section{DiE STUFEN DES BEWEISES UND IHRE PARALLELE STRUKTUR}

Zunächst sollte festgestellt werden, dass in diesem Text verschiedene Stufen des Beweises unterschieden werden können, die von unten nach oben Schritt für Schritt erklommen werden. Das bedeutet keineswegs, dass diese Stufen faktisch voneinander isoliert existieren können. Diese Trennung der Stufen bezieht sich ausschließlich auf die abstrakte Charakterisierung des Beweises. Meiner Betrachtung nach können mindestens vier Stufen unterschieden werden. ${ }^{33}$ Mit anderen Worten fordert die Welt eine Ausweisung des Bewusstseins, die in vier Stufen getrennt betrachtet werden kann:

a) das eine Welt ausweisende, «formal allgemeine» Bewusstsein;

$\left.\mathrm{a}^{\prime}\right)$ das eine Welt ausweisende, «menschliche», «leibliche», ${ }^{34}$ «animalische ${ }^{35}$ Bewusstsein (mit der Räumlichkeit und der «Freiheit» ${ }^{36}$ der Orientierung in ihr);

31

32

33 tivität handelt, und die «Oberstufe», wo es sich um mehrere Subjektivitäten unter einer «akkordierten "Gemeinschaft"» handelt (Siehe Hua XXXVI. S. 143-144).

34 Siehe Hua XXXVI. S. 132. Auch Hua XXXVI. S. 133: «Im Raum ist unter anderen Dingen ein Leib, und mit dem bin ich eins. Ich, als reines Ich und zugleich als vermögliches Ich, bin an ihn gebunden, bilde mit ihm eine reale Einheit».

Ibid. Über die «Freiheit» in der Räumlichkeit wurde oben bereits gesprochen. 
b) die eine Welt ausweisenden leiblichen Subjektivitäten (mit der «Einfühlung» $\left.{ }^{37}\right)$;

$\mathrm{b}^{\prime}$ ) die eine Welt im «Akkord $»^{38}$ ausweisende «Gemeinschaft» $»^{39}$ von mehreren Subjektivitäten, darin das Auf- und Abtreten der Subjektivitäten $\left.(« \text { Geburt }\rangle^{40}, \ll T o d »^{41}\right)$ berücksichtigt sind.

Die letzte Stufe des Beweises ist dadurch gekennzeichnet, dass hier unterschiedliche Subjektivitäten miteinander verbunden sind und eine harmonische Gemeinschaft konstituieren, die ein und dieselbe Welt direkt oder indirekt ausweisen können.

Auf den ersten Stufen a), a') handelt es sich um die Einheit einer wirklichen Subjektivität, die eine Welt wirklich ausweisen kann, auf den zweiten Stufen b), b') handelt es sich um die harmonische Gemeinschaft mehrerer real möglicher Subjektivitäten, die ein und dieselbe Welt durch die Einfühlung real möglich ausweisen können.

Dazu könnte eine parallele Struktur zwischen den beiden Stufen betrachtet werden - zwar nicht faktisch, aber rein formal strukturell gesehen.

Auf den ersten Stufen kann das «Ich» das Nebeneinander oder den Wechsel der verschiedenen erkennenden (verträglichen oder unverträglichen) Iche ${ }^{42}$ sein, die in der Wirklichkeit nur als ein einziges wirkliches Ich existieren.

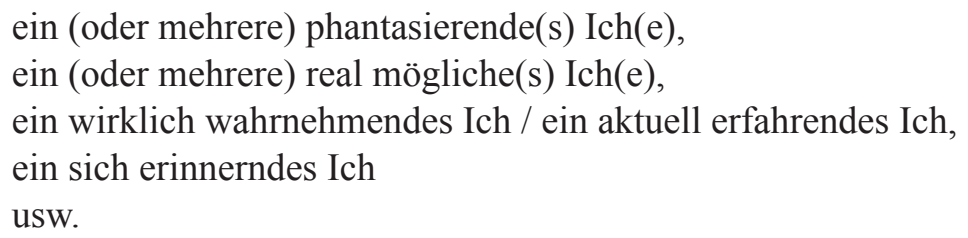

37 Siehe Hua XXXVI. S. 134. Die «Einfühlung» ist nicht trennbar von der «Leiblichkeit»: Erfahrungen in sich nacherfahren zu können, ist «nur denkbar als Einfühlungsverhältnis» und fordert, dass die Subjekte «animalische Existenz» sind.

38 Hua XXXVI. S. 143. «Die Welt ist geschaffen, d. h. eine Subjektgruppe gewinnt gemäß einer bestimmten Regelordnung des Akkords bewusstes Leben».

39 Siehe Hua XXXVI. S. 144.

40 Husserl meint «Geburt» als a priori, insofern das Bewusstsein leiblich sein muss, insofern es «in der Welt» sein muss: «<... die Erfahrungsapperzeptionen [weisen] auf eine Genesis a priori zurück <..> Jedes Subjekt hat seine Geburt, und das... aus... apriorischen Gründen, wenn es nämlich die Bedingungen eines "Subjekts in einer Welt" erfüllen soll» (Hua XXXVI. S. 141).

41 Hua XXXVI. S. 143. «Jede Subjektivität hat eine Lebensperiode vorweltlicher Subjektivität, dann eine Lebensphase in der Welt, und dann eine Phase nachweltlicher Subjektivität, die des Todes».

42 Siehe auch: Hua VIII. S. 90; Hua XXV. S. 276; Hua XXXVII. S. 104. 
Hier ist das wirkliche Ich das Beherrschende unter anderen, worauf alle Ausweisungen der (Quasi-) Wahrnehmungen konvergieren und das unter diesen Ausweisungen einige wählen und andere ausschließen kann.

Das konvergente Schema könnte auf den zweiten Stufen als das «Wir» bezeichnet werden:

das wirkliche Ich (das Ich, worauf alle oben gesehenen Iche konvergieren), ein Anderer, noch ein Anderer usw.

Hier ist der Konvergenzpunkt aller Ausweisungen das wirkliche Ich, das alle Ausweisungen der Anderen durch synthetische Konstruktion beherrscht. Für beide Stufen gäbe es die parallele Struktur, nicht im faktischen Sinne, sondern insofern als das wirkliche Ich als Konvergenzpunkt durch verschiedene Ausweisungen die Einstimmigkeit und die Einheit dieser Ausweisungen geben kann. Mit andern Worten sind Einheit und Wirklichkeit in beiden Stufen gefordert. Der Husserlsche transzendentale Idealismus besagt also: die Möglichkeit einer Welt (einer Natur, eines Dings usw., nicht bloß ideale Möglichkeit) fordert die Wirklichkeit und die Einheit des/der Erkennenden, sowohl für eine Subjektivität als auch für die Gemeinschaft mehrerer Subjektivitäten. ${ }^{43}$

So könnte der Beweis, mit dem wir uns im nächsten Abschnitt beschäftigen werden, für diese beiden Stufen, formal gesagt, gleichzeitig gültig verwendet werden.

\section{Der Beweis Des transzendentalen Idealismus}

\section{Die UNäquiVALENZ ZWISCHEN WELT UND BEWUSSTSEIN}

Die Eigenschaft der Intentionalität, des Grundbegriffes der Husserlschen Phänomenologie, spiegelt sich über die Korrelation zwischen der Gegenständlichkeit und dem Bewusstsein. Im Unterschied zu Descartes stellt Husserl das «Sinnliche» nicht infrage, weil es als «originär Gegebenes» zweifellos ist,${ }^{44}$ wie

43 Aber in den Texten Nr. 5-7 scheint die Frage über die Zukunft offengelassen zu sein. Husserl selbst schreibt in einer Fußnote: «Da wir für die Vielheit der Subjekte und ihrer Bewusstseinsentwicklungen keine umspannende objektive Zeitform haben, so fragt es sich, was da "Zusammenhang", intersubjektive Koordination, "neues Eintreten” etc. besagen soll!!» (Hua XXXVI, S. 144).

44 Descartes stellt das «Sinnliches» infrage, während Husserl es schlechthin als Quelle der Erfahrung nimmt: «Übernehme ich seinen [= Descartesschen] Ausgang von der Kritik der Sinnlichkeit, so modifiziere ich sie $<\ldots>$ dadurch, dass ich die "sinnliche Erfahrung" als meines ego 
es schon oben als das Wesen der Wahrnehmung dargestellt wurde. Der Grundgedanke besteht also darin, dass, wenn es eine mögliche Gegenständlichkeit gibt, es eine ihr entsprechende Ausweisung geben muss, also das sie ausweisende Erkennende. Denn wenn es einen Pol der Intentionalität gibt, muss es auch ihren anderen Pol geben. Sehen wir uns diese Korrelation näher an:

für eine mathematische Gegenständlichkeit - einen mathematisch ausweisenden Erkennenden, ${ }^{45}$

für eine ideal mögliche Gegenständlichkeit — einen ideal Mögliches ausweisenden Erkennenden,

für eine fiktive Gegenständlichkeit — ein quasi-wahrnehmendes fiktives Ich,

für eine real mögliche Gegenständlichkeit - einen real Mögliches ausweisenden Erkennenden,

für eine wirkliche Gegenständlichkeit — einen Wirkliches ausweisenden Erkennenden. ${ }^{46}$

Was beachtet werden muss, ist, dass diese Korrelationspaare nicht immer miteinander verträglich sind. Nehmen wir das Beispiel der Fiktion. Die Fiktion hat den Charakter der Quasi-Wahrnehmung: «Die Fiktion fingiert mir ein Ding als seiend, und zunächst nicht nur überhaupt als seiend, sondern zugleich als wahrgenommen seiend». ${ }^{47}$ Das bedeutet, dass auch die Fiktion

Bewusstsein betrachte und einer intentionalen Auslegung unterziehe, in dem mir irgendein Reales und schließlich die Welt, das für mich seiende Universum raumzeitlicher Realitäten, allen zur originären Gegebenheit und überhaupt zur eigenen Erfahrung kommt und kommen kann als seiend und soseiend» (Hua XXXVI 130). Für Husserl ist diese Weise der Erscheinung des Sinnlichen nicht zufällig, sondern «wesensnotwendig»: «Überlegen wir in eidetischer Einstellung das reine Wesen der Wahrnehmung, so erkennen wir, dass die Transzendenz des Wahrnehmungsdinges, des sinnlich erscheinenden, so wie es da erscheint, keine zufällige, sondern eine wesensnotwendige ist...» (Hua XXXVI. S. 90).

Die mathematische Existenz fordert nur das mathematisch ausweisende Erkennende, und fordert nicht, dass dieses Erkennende wirklich existiert: «Die mathematische Existenz von Zahlen, Mannigfaltigkeiten etc. fordert mit der idealen Möglichkeit der einsichtigen Ausweisung nicht die wirkliche Existenz eines Bewusstseins, das unmittelbar oder mittelbar auf Mathematisches bezogen oder zu beziehen ist» (Hua XXXVI. S. 74. Siehe auch Hua XXXVI. S. 120). «Jeder individuelle Gegenstand, den wir als existierend ansetzen, fordert alsbald die wirkliche Existenz eines Bewusstseins» (Hua XXXVI. S. 74). «Wirkliche Existenz eines Dinges... fordert ein inhaltlich ausgezeichnetes aktuelles Ich, ein wirklich existierendes Bewusstsein mit wirklichen Erfahrungen und Erfahrungsthesen» (Hua XXXVI. S. 78. In diesen Sätzen sind die beiden Glieder der Korrelationen äquivalent).

Hua XXXVI. S. 113.

Horizon 1 (2) 2012 
die Orientierung für die verschiedenen Abschattungen fordert. Wie oben beim Wesen der Wahrnehmung dargelegt wurde, hat diese Quasi-Wahrnehmung etwas von Quasi-Leiblichkeit, aber nicht von der Leiblichkeit dieses aktuell erfahrenden Ichs in der Wirklichkeit. Das phantasierend quasi-wahrnehmende Ich kann nicht mit dem wirklich wahrnehmenden Ich dasselbe sein. Husserl zeigt das am Beispiel des einen Zentauren phantasieren Ichs ${ }^{48}$ oder des eine Nixe phantasierenden Ichs: «...ich kann wohl nicht ein Ding fingieren, ohne es als ein wahrgenommenes eines wahrnehmenden Ich fingiert zu haben, und wenn ich dabei auch mich an die Stelle dieses Ich phantasiere, so bin ich dabei eben mögliches Ich, nicht aber das wirkliche Ich» ${ }^{49}$ Damit wird festgestellt, dass das phantasierend quasi-wahrnehmende Ich mit dem aktuell erfahrenden wirklichen Ich unverträglich ist. Die Korrelation in der Sphäre der Fiktion und diejenige in der Sphäre der Wirklichkeit sind miteinander inkompatibel.

Aber unter den oben angegebenen Korrelationen sind nicht alle Erkennenden als Ich unverträglich. Beispielsweise müssen das wirkliche Ich und das real mögliche Ich verträglich sein, wenn dieses real mögliche Ich in der Aktualisierung zum wirklichen Ich kommt, ohne von diesem ausgeschlossen zu werden. Aber dieses real mögliche Ich muss das einzige sein und alle anderen real möglichen Iche müssen damit ausgeschlossen werden.

Was infrage gestellt werden soll, ist der Übergang dieser verschiedenen Korrelationen, obwohl diese nicht immer oder meistens miteinander unverträglich sind. Mit anderen Worten: Warum fordert die Möglichkeit einer Welt (einer Natur) die Wirklichkeit des Erkennenden oder der Erkennenden, obwohl es diese Unäquivalenz der beiden Glieder der Korrelationen gibt? Nicht nur eine wirkliche Welt fordert die Wirklichkeit eines Bewusstseins, sondern auch eine mögliche Welt, sei es möglich, ohne wirklich zu sein (Möglichkeit Wirklichkeit), sei es nicht nur möglich, sondern auch wirklich (Möglichkeit + Wirklichkeit). Die mögliche Welt als mögliche fordert ein aktuell erfahrendes wirkliches Bewusstsein. Das ist das, was Husserl mit der These des transzendentalen Idealismus sagt, wie wir in der Einführung gesehen haben. Hier liegt die Unäquivalenz zwischen der Welt und dem Bewusstsein. Die oben gesehenen Korrelationen bewahren also diese Korrelationen im strengen Sinne nicht. Die Eigentümlichkeit dieses Beweises besteht in dieser Unäquivalenz. Wie könnte diese Unäquivalenz verstanden werden?

48 


\section{DER KERN DES BEWEISES}

Um diese Frage aus der Nähe zu betrachten, muss eine Stelle, die im Text Nr. 7 steht, ${ }^{50}$ als äußerst wichtig angesehen werden. Der Beweis, der hier entwickelt wird, beginnt auf den zweiten Stufen des Beweises, die die Mehrheit der Subjekte betreffen, aber inhaltlich bezieht sich die in Frage kommende Stelle doch auf die erste Stufen, die ein Subjekt betreffen. Meines Erachtens könnte die parallele Struktur, die im Absatz I-3 betrachtet wurde, durch dieses Ineinander der Stufen des Beweises erklärt werden.

Hier werden wir uns darauf konzentrieren, wo Husserl in diesem Beweis zuerst eine mögliche Natur A setzt, wie wir in der Einführung bereits gesehen haben: «Die Möglichkeit der Natur setzt voraus oder schließt idealiter ein die Möglichkeit einer Erkenntnis dieser Natur. Die letztere Möglichkeit fordert, das wäre dann weiter zu zeigen, nicht die bloß logische Möglichkeit von "Menschen in der Natur", sondern wirkliche Existenz derselben». ${ }^{51}$

Warum musste von der Möglichkeit einer «Natur» ausgegangen werden? Weil, erstens, von einem der beiden Glieder der Korrelation ausgegangen werden muss. Wenn aber, zweitens, von einer Subjektivität ausgegangen wird, kann dem Beweis des transzendentalen Idealismus nicht gefolgt werden. Um einen Beweis liefern zu können, darf nicht die Subjektivität am Anfang vorausgesetzt werden.

Hier heißt «möglich» (von der «Möglichkeit der Natur»), dass entweder das A möglich ist, ohne wirklich zu sein, oder das A nicht nur möglich, sondern auch wirklich ist. Husserl fängt wie folgt an: «Sei A eine Welt oder überhaupt ein nicht-ideales Sein, also ein matter of fact, dann haben wir zwei offene Möglichkeiten a priori: A existiert nicht, obschon es möglich ist, und A ist nicht nur möglich, sondern existiert auch». ${ }^{52}$

Zwei Fälle werden hier betrachtet:

A: Möglichkeit - Wirklichkeit

A: Möglichkeit + Wirklichkeit

Wenn A möglich und wirklich ist, ist die Ausweisung der A erkennenden Subjektivität möglich und wirklich; das kann ohne Weiteres akzeptiert werden. Wenn A nicht existiert, d. h. wenn A möglich ist, ohne wirklich zu sein, ist die Ausweisung des A nicht möglich. Warum? Denn die «Nicht-Existenz des A» ist jetzt wirklich, und diese Nicht-Existenz muss ausgewiesen werden

50 Hua XXXVI. S. 138-140.

51 Ibid. S. 135. Oben zitiert. Siehe die Fußnote 5 dieses Aufsatzes.

52 Ibid. S. 138.

Horizon 1 (2) 2012 
können. Wenn die Ausweisung des A gleichzeitig möglich ist, entsteht ein Widerspruch im Vollzug: «Existiert A nicht, so ist sie [= Seinsausweisung] unmöglich. Denn, wäre sie möglich, $<\ldots>$ so wäre die rechtmäßige Erkenntnis verträglich mit der Nichtexistenz des Erkannten, was ein Widerspruch ist». ${ }^{53}$

Die Existenz des A ist unverträglich mit der Nicht-Existenz des A. D. h. wenn A existiert, ist die Ausweisung notwendig möglich, und wenn A nicht existiert, ist die Ausweisung des A notwendig unmöglich. Die Existenz des A definiert sich überhaupt nur durch die Möglichkeit / Unmöglichkeit der Ausweisung. Das entspricht der These des Anfangs von Text Nr. 5: «Der Satz "A existiert" und der Satz "Es ist ein Weg möglicher Ausweisung der Existenz des A zu konstruieren", "Es besteht die ideale und einsehbare Möglichkeit solcher Ausweisung" sind Äquivalenzen». ${ }^{54}$

Dass die Möglichkeit der Existenz des A abgelehnt wird, bedeutet, dass sie eine «reale» Möglichkeit ist. Es muss hier daran erinnert werden, dass für ein mögliches, aber nicht wirkliches A zwei Erkenntnisse gedacht werden können. Auf der einen Seite die bloß logisch mögliche Erkenntnis, auf der anderen Seite die nicht bloß logisch mögliche Erkenntnis. Durch die bloß logisch mögliche Erkenntnis können unendlich viele Ausweisungen des A gedacht werden, sofern sie einander nicht widersprechen. Aber die nicht bloß logisch mögliche Erkenntnis muss abgelehnt werden, wenn sie nicht wirklich ist. Sie ist eine reale Möglichkeit, wie oben gesehen wurde: «...die Unwirklichkeit eines möglichen A schließt $<\ldots>$ die Möglichkeit der Erkenntnis aus.... Somit ist die hier ausgeschlossene Möglichkeit, die zur Existenz des A gehört, sicher nicht $<$ die $>$ bloß logische Vorstellbarkeit. Sie ist "reale" Möglichkeit...». ${ }^{55}$

Wie im Absatz I-2 näher betrachtet wurde, wird die reale Möglichkeit mit der Wirklichkeit gebunden. Da das wirkliche A vom wirklichen Bewusstsein ausgewiesen werden kann, muss die reale Möglichkeit von diesem wirklichen Bewusstsein mitgesetzt werden können. Daraus folgt, dass es sich immer um eine «wirkliche» Erkenntnis handelt, wenn A real möglich ist, egal ob A existiert oder nicht. So kommt der Schluss des Beweises zu dem Ergebnis: «Die Existenz eines möglichen A (aber eines zufälligen, dessen Nichtexistenz also "logisch" offen ist) fordert die Existenz eines wirklichen Subjekts...». ${ }^{56}$ 
Diese bis jetzt betrachtete Textstelle ist meines Erachtens der Kern des Beweises. Erst am Ende dieser Stelle sagt Husserl, dass sein Beweis «lückenlos» sei. ${ }^{57}$ Nun könnte man sagen, dass die These des transzendentalen Idealismus, die am Anfang von Text Nr. 5 beschrieben wird, bewiesen sei.

\section{VERSCHIEDENE EINWENDUNGEN UND WIDERLEGUNGEN}

Das, was diesem Beweis kohärent zugrunde liegt, ist, wie wir bis jetzt gesehen haben, die Zusammengehörigkeit der Wirklichkeit und der AusschlieBung, die auf dem Satz vom Widerspruch beruht. Ist das A wirklich, müssen andere (reale) Möglichkeiten des A ausgeschlossen werden. Werden alle anderen Möglichkeiten des A ausgeschlossen, ist das A wirklich. Die Wirklichkeit bedeutet also Einheit und Ausschließung. Mit anderen Worten gibt es eine einzige Gemeinsamkeit der Zusammenhänge der Wirklichkeiten und die damit verbundenen verschiedenen realen Möglichkeiten. Aber die bloß idealen Möglichkeiten sind viel zahlreicher als die realen. ${ }^{58}$

Gefragt wird, wie es zu einer «Fixierung» eines Ichs ${ }^{59}$ unter unendlich vielen Möglichkeiten oder wie es zu einer «doxischen Setzung» ${ }^{60}$ eines Ichs kommt. Dieses Ich kann die Einheit für die Zusammenhänge der Ausweisungen darstellen. Wenn einmal das wirkliche Ich in der Aktualisierung fixiert ist, löst es alle anderen realen Möglichkeiten auf, denn alle anderen Möglichkeiten werden ausgeschlossen. Aber wie konnte «ein» Ich — wir haben das bis jetzt unhinterfragt vorausgesetzt — in einer Leiblichkeit durch eine mögliche Natur, die nicht existieren kann, fixiert werden?

Husserl versucht selbst, solche Einwendungen gegen seinen eigenen Beweis aufzustellen.

a. Die Ausschaltung oder die Durchstreichung eines Ichs, ${ }^{61}$

b. Die Zerstreuung (Spaltung, Zerstückelung) eines Ichs (z. B. eines verrückten Ichs ${ }^{62}$ oder die Disharmonie mehrerer Ichs. ${ }^{63}$

57 Ibid.

58 Siehe Abbildung 2.

59 «Fixieren wir ein Ich...» (Hua XXXVI. S. 117).

60 Ibid.

61 «Schalten wir die Setzung der gegeben Welt zunächst aus und zugleich auch die Setzung unseres erfahrenden Ich mit seinen bestimmten Erfahrungen» (Hua XXXVI. S. 117); oder: «...wenn wir alle Ich... gestrichen denken würden...» (Hua XXXVI. S. 121).

62 Siehe Hua XXXVI. S. 144.

63 Darüber spricht Husserl nicht explizit in diesen Texten, aber die Frage nach der Geburt und dem Tod, die hier skizziert wird, eröffnet dieses Problem der Disharmonie mehrerer Iche. 
Diese Versuche der Durchstreichung des Ichs oder der Zerstreuung des Ichs (des verrückten Ichs) schließen immer die Folgerung ein, dass das Ich nicht zu streichen oder zu zerstreuen ist, weil es für die mögliche Welt (oder die mögliche Natur) eine mögliche Ausweisung als «ein» letztmaliger Pol geben muss. Nehmen wir ein Beispiel: «Eine Naturwelt mit unvernünftigen Wesen der Art, wie es Verrückte sind, ist nur möglich, wenn die Unvernünftigen in jener uns vertrauten unvollkommenen Gemeinschaft mit vernünftigen Wesen auftreten, die diese Unvernünftigen in die Welt einordnen können. Also die Welt bezogen auf notwendig normale Subjekte». ${ }^{64}$

Hier könnte festgestellt werden, dass die Unvernünftigkeit, bzw. die Verrücktheit, immer auf eine Gemeinschaft vernünftiger Subjekte zurückbezogen werden muss, die die Welt - oder die Natur - letztlich ausweisen kann.

In einer Randbemerkung im $\S 49$ der Ideen I, die von Schuhmann veröffentlicht wurde, stellt sich Husserl noch einmal diese Frage, ${ }^{65}$ und erwähnt «ein anderes Ich», das statt des Ichs real möglich die Möglichkeit der Welt ausweisen kann. Wenn das verrückte Ich eine Unstimmigkeit der Welt erfahren kann, setzt dieses Ich schon die Möglichkeit der stimmigen Welt voraus. ${ }^{66}$ Aber da dieses Ich diese Möglichkeit nicht anerkennen kann, soll derjenige, der sie anerkennen kann, ein anderes Ich sein, das dieses Ich als real möglich erfahren kann. ${ }^{67}$ Das entspräche dem oben gezeigten Zitat im Beweis des transzendentalen Idealismus. Hier gibt es immer einen Verweis auf ein vernünftiges real mögliches Ich.

\section{ZUM SCHLUSS - DIE IDEE IM KANTISCHEN SINNE UND DER SATZ VOM WIDERSPRUCH}

Wirklichkeit bedeutet Einheit. Daraus ergibt sich eine Frage: Fußt die letztmalige Ausweisung als der beherrschende Punkt der Zusammenhänge der verschiedenen Ausweisungen auf der einzigen Gegenwart ohne Beweglichkeit der

64 Ibid.

65 Husserl E. Ideen zu einer reinen Phänomenologie und phänomenologischen Philosophie, Erstes Buch, 2. Halbband, Ergänzende Texte (1912-1929) / hrsg. von Karl Schuhmann. Den Haag: Nijhoff, 1976, Hua III/2. S. 498. «Es ist möglich, daß meine Erfahrung Ausweisungen für eine Erfahrungswelt, <...> unmöglich machen. Aber darum kann doch sehr wohl eine mir unzugängliche Welt möglich sein und die Welt, die wirklich ist, nur daß ich verrückt bin...».

66 Ibid. «...wenn ich das anerkennen soll, [dass ich verrückt bin,] muß ich die Möglichkeit einer Welt einsehen können».

67 Ibid. «Entweder ich bin es selbst, der in seinem reinen Eigenwesen diese Möglichkeit [= reale Möglichkeit der Welt] erkennen kann, oder es <ist> ein anderes Ich etc. Dieses andere kann nicht für mich leere Möglichkeit sein, es müßte selbst in meinem Erleben begründet und begründbar sein»». 
Zerstreuung, Spaltung oder Durchstreichung? Denn der Widerspruch und die Ausschließlichkeit, die im Widerspruch fundiert ist, könnten sich nur dann ergeben, wenn diese Einzigkeit der Gegenwart ohne Verschiebung oder Aufschub versichert wird, um die beherrschende letzte Ausweisung zu vollziehen. ${ }^{68}$

Die Kritik der Bilder- und Zeichentheorie, die oben betrachtet wurde, setzt auch diese einzige Gegenwart, d. h. die Gleichzeitigkeit der letzten Ausweisungen (der Ausweisung des Ichs und derjenigen des gespalteten Ichs) voraus. Weil die Spaltung des Ichs in infinitum unsinnig ist, wenn diese Gleichzeitigkeit gefordert ist. Was kann aber eine solche Gleichzeitigkeit sichern?

Wie im Absatz I-2 dargestellt wurde ${ }^{69}$, kann die Wirklichkeit (das Ding) als eine Idee im Kantischen Sinne betrachtet werden, mit anderen Worten kann die Einheit, die nichts anderes als die Wirklichkeit ist, auch als diese regulative Idee, die immer unbestimmt bleibt und die sich immer weiter vollziehen lässt, angenommen werden. D. h. die Einzigkeit der letzten Ausweisung oder die Gleichzeitigkeit der letzten verschiedenen Ausweisungen (wie im Fall des Widerspruchs) müsste auch die Idee im Kantischen Sinne sein. In Absatz I-3 dieses Aufsatzes wurde gezeigt, wie Husserl die Frage nach der Zukunft offenlässt, das Problem der Geburt und des Todes erwähnend. ${ }^{70}$ Müsste die Idee im Kantischen Sinne nicht gegen Spaltungen oder Zurücknahmen offenbleiben? Müsste die Gegenwart nicht beweglich und immer für die Zerstreuung geöffnet sein? Gegen eine einzige Gegenwart könnten verschiedene Einwendungen gemacht werden. Zeigt das Phänomen des Traumas nicht dasjenige, das von allen Zusammenhängen der Ausweisungen vergessen wurde? Oder die Toten, an die sich niemand erinnern kann, und deren Existenz von niemandem, weder direkt noch indirekt, ausgewiesen wird, von niemandem, auf den ich mich aktuell erfahrend beziehe? In dieser Hinsicht könnte Jacques Derridas Lektüre Husserls, die die Idee im Kantischen Sinne sehr betont, bedeutsam sein. In seinem Text «Gewalt und Metaphysik», wo er zu zeigen versucht, dass

68 In Ideen I vollzieht Husserl die Reduktion der Logik, von der "Ausschaltung der reinen Logik als mathesis universalis» sprechend. Aber selbst wenn die Logik ausgeschaltet wird, erweitert sich diese Ausschaltung nicht bis zum Satz vom Widerspruch. «Die Phänomenologie ist nun in der Tat eine rein deskriptive, das Feld des transzendental reinen Bewusstseins in der puren Intuition durchforschende Disziplin. Die logischen Sätze, auf die sich zu berufen sie je Anlaß finden könnte, wären also durchaus logische Axiome, wie der Satz vom Widerspruch, deren allgemeine und absolute Geltung sie aber an ihren eigenen Gegebenheiten exemplarisch einsichtig machen müßte. Die formale Logik und die ganze Mathesis überhaupt können wir also in die ausdrücklich ausschaltende $\varepsilon \pi \circ \chi \eta ்$ einbeziehen...» (Hua III/1. S. 127, § 59).

Siehe oben: Fußnote 28 dieses Aufsatzes.

Siehe Fußnote 43 dieses Aufsatzes. 
die Husserlsche Phänomenologie nicht so weit von dem, was Levinas versucht, entfernt ist, erwähnt Derrida diese Idee im Kantischen Sinne als das, was eine absolute Offenheit für die Alterität erlaubt.

«...[D]ie Idee im kantischen Sinne [bezeichnet] das unendliche Überborden eines Horizonts, der ...niemals selbst Gegenstand werden oder durch gegenständliche Anschauung ausgefüllt und ausgeglichen werden kann. $<\ldots>$ Wenn die Unendlichkeit des Husserlschen Horizontes die Gestalt der unbestimmten Öffnung besitzt, ...ist das nicht das, was ihn am sichersten vor jeder Totalisierung, vor der Illusion der unmittelbaren Präsenz eines erfüllten Unendlichen bewahrt $<\ldots>$ ? $\rangle^{71}$

Was die Totalisierung aus dem einzigen Ich aufschiebt, ist der Aufschub, der jedes Mal den Pol als Idee im Kantischen Sinne verschiebt. Der Aufschub, der in der Idee im Kantischen Sinne wohnt, musste auch die Etablierung des Satzes der Identität, des Satzes vom Widerspruch, des Satzes vom ausgeschlossenen Dritten aufschieben. Von Gödel erwähnend, ${ }^{72}$ weist Derrida darauf hin, dass das, was Husserl zu entdecken versucht, nicht weit von «tertium datur» ${ }^{73}$ vor «tertium non datur» als Satz vom ausgeschlossenen Dritten entfernt ist.

Der Schluss, der daraus gezogen werden kann, ist der Folgende: Der Aufschub in der Zeitlichkeit, worin die Idee im Kantischen Sinne immer unbestimmt bleibt, muss berücksichtigt werden, weil er die Gleichzeitigkeit immer verschiebt und dadurch nicht erlaubt, dass sich der Satzes vom Widerspruch oder der Satz der Identität etablieren. Dadurch könnte auch die Alterität, die das erkennende Ich nie ausweisen kann, erlaubt werden.

In den betrachteten Schriften Husserls selbst könnte auch eine Möglichkeit für eine solche Weiterführung gesehen werden, wenn der oben betrachtete Text über die Verrücktheit (Randbemerkung des $§ 49$ der Ideen I) etwas anders gelesen wird bzw. wenn das im Zitat genannte «andere Ich», jenseits aller möglichen Ausweisungen in der Gegenwart, die reale Möglichkeit einer Welt angeben könnte, die das Ich nie ausweisen kann. Diese Antwort könnte nicht ausreichend sein, wenn die zwei Stufen des Beweises, die sich auf mehrere Subjektivitäten beziehen, die ersten Stufen, die sich mit einer Subjektivität befassen, als Voraussetzung benötigten. Aber wie oben erwähnt wurde, sind diese Stufen faktisch nie getrennt. Diese Randbemerkung zeigt uns eine weitere Möglichkeit des Beweises des transzendentalen Idealismus.

71 Derrida J. Die Schrift und die Differenz, Frankfurt am Main: Suhrkamp, 1972. S. 183 184; L'écriture et la différence, Paris: Seuil. S. 177.

72 Derrida J. [Die Einführung zu Husserls Text :] L'Origine de la géométrie, Paris: PUF, 1962. S. 39.

73 Op.cit. S. 40. 


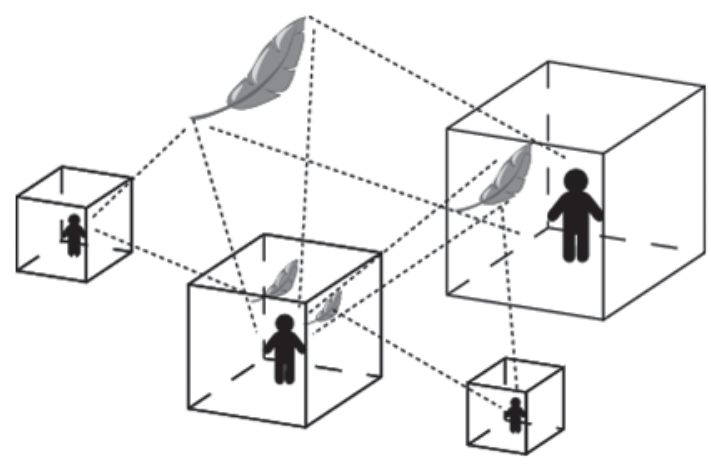

Abbildung 1

Die Kritik der BiLder- Und Zeichentheorie (I-1-2)

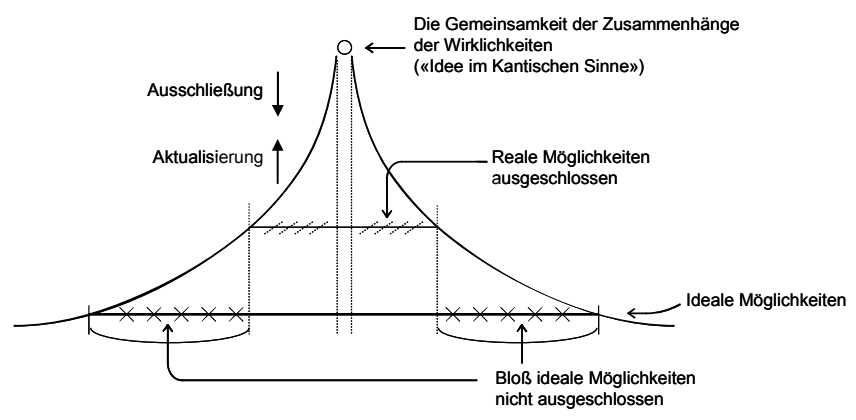

Abbildung 2

Die reale Möglichkeit / die Wirklichkeit und die bloß idealen Möglichkeiten (I-2)

\section{A UTHOR}

NAGASAKA MAsumi — Ph.D Student at Kyoto University (2007) as well as a member of the joint doctoral program of the University of Wuppertal and University of Toulouse II (2009).

НАГАСАКА Масуми - аспирантка (Ph.D. student) Университета Киото (Япония), аспирантка (Ph.D. student) университетов Тулузы и Вупперталя.

E-Mail: summer_en_tunisie@yahoo.fr 